The track that the sphere made on the surface would be likely to be from the direction of the bus and that track is thus also not on the available panorama. The other possibility, that the bus is not visible because it sank out of sight, but that on the other hand on the same surface the sphere left no visible track, is elearly most unlikely. The experimentation initially proposed then probably included a study of the visible track, and it was only the unfortunate angle in which the sphere came to rest that made this impossible.

The subsequent movement of Luna 9. It appears that between the picture-taking sequences, a movement occurred of unknown origin in which the camera position was changed by several inches. It was suggested in the Press that the experimenters involved had no knowledge of the origin of this movement and did not attribute it to any activity within the vehicle.

Earthquakes are clearly outside the range of reasonable explanations since a degree of such activity would be required that would have shown up in other ways.

On any dry, terrestrial ground, and in the absence of wind, it would seem very improbable that an object after coming to rest would make significant movements in a period of days. The observation must thus be taken to be a very significant one that implies properties of the lunar surface quite unlike those of terrestrial surfaces.

In whatever position the vehicle came to rest and whatever stresses, such as by spring-loaded panels, were then in effect, it would be necessary to discover some process with a time constant in the range of hours, to allow the movement to be triggered off. The immediate dynamical time constants of the settling would be in the neighbour- hood of seconds only. Plastic time constants in dry rock are many orders of magnitude too long. Thermal heating and cooling could have the right time constants, but the displacements resulting are so small that one would need to suppose the vehicle to be extremely delicately poised for such effects to trigger off a substantial movement.

It is thus quite a puzzle to discover some effect that would have been going on for a period of hours after the landing which, when it reached a certain stage, allowed a sudden resettling of the vehicle. Time constants of the same nature as those in an hour-glass may provide an explanation. If the material of the lunar ground were composed of small rock particles, slightly adhering to each other, it would be possible for there to be a lot of sub-surface hollow spaces. In particular, many small secondary impacts at rifle bullet speeds would make long tunnels at all angles through such a material. The structure that results would be the statistical equilibrium between the compacting effects of some types of impact and the tunnel-digging effects of others. On such a surface it would then not be too unlikely for the added weight of the vehicle to result in many little slides and collapses in internal cavities, which gradually reduce the strength of the ground and eventually let it settle. The time constants, as in an hour-glass, would be those of the dynamical time constants of falling grains but multiplied by the number of grains that had to fall in sequence to produce the effect.

While this explanation can be taken as a suggestion only, the fact that the ground tends to give way after some period of time under an additional load has probably to be taken seriously.

\title{
SIGNIFICANCE OF INCLUSIONS IN CARBONACEOUS METEORITES
}

$\mathrm{T}$ HE possibility has frequently been suggested and discussed that all meteorites which have become available for observation have originated from the same parent body, or from a comparable set of parent bodies, of asteroid dimensions. Such a theory is supported by hitherto observed instances of interbrecciations between: siderite-pallasite; siderite-mesosiderite; siderite-chondrite; siderite-achondrite; mesosiderite-chondrite; mesosiderite-achondrite; chondrite-achondrite (for a summary see refs. 1 and 2 ).

That such a common origin exists has not yet been proved through actually observed associations of those meteorites which could be included in the 'main carbonrich groups', with more than 0.2 per cent carbon (namely, $(A)$ carbonaceous chondrites; $(B)$ ureilites; $(C)$ enstatite chondrites).

The thirty or so carbonaceous chondrites which have so far been recorded have been sub-divided by Wiik ${ }^{3}$ into three types; the carbon + water + sulphur ranging between 24 and 30 per cent for 'type I'; 12 and 24 per cent for 'type II'; 3 and 12 per cent for 'type III'. Recent investigations have shown ${ }^{4}$ that 'type $I$ ' stones consist of more than 90 per cent low-temperature ground mass of hydrated silicates and carbonaceous matter, of grain-size less than $l \mu$, in which are embedded globular or rounded particles, varying in diameter from 5 to $50 \mu$, of magnetite, glass, olivine, troilite and other high-temperature minerals. I have interpreted ${ }^{4,5}$ these to be 'microchondri', that is, solidified 'fog' particles from an incandescent cloud, as often they reveal exsolution cores. In the 'type II' stones, the ground mass is reduced to about 60 per cent and larger chondrules, that is, 'raindrops', are present in addition to the 'microchondri'. Most of these are, however, of the 'bunch of grapes' type, and still reveal the original microchondrules. These apparently became compacted in a viscous or sticky state. Within 'type III' stones the ground mass is reduced to about 35 per cent, and the majority of the chondrules are 'fully coalesced'; the latter stones grade into the non-carbonaceous chondrites. The three hitherto known ureilites contain between 0.5 and 1 per cent carbon, and they consist of what appear to be squashed single crystals or granular aggregates (possibly of 'microchondrules') of silicates, with concentrations of carbonaceous matter and diamonds along the grain boundaries. The ten enstatite chondrites contain 3-10 per cent carbon + water + sulphur, and, roughly speaking, have a petrographical structure similar to the 'type III' carbonaceous stones or the ordinary chondrites. More detailed descriptions of the carbonrich groups of meteorites are to be found elsewhere $2,4,6,7$.

This article describes some recently observed examples of inhomogeneities, inclusions and interbrecciation, in meteoritic materials of which at least one sample belongs to the foregoing 'carbon-rich' groups.

\section{(1) Orgueil}

\section{Inclusions}

Inclusion $A$. In the course of preparation of material for analytical work during 1962 , I carefully separated some $3 \mathrm{~g}$ of the stone, obtained from a 20 -g crust-less fragment from the Museum in Paris. An unusually hard, angular inclusion was found, which on analysis revealed a rather higher percentage of troilite plates and magnetite microchondrules than was present in the rest of the stone; however, the petrographical structure remained within the range of the 'type I' stones. An essentially similar type of angular inclusion was observed in a thin section of the Orgueil meteorite ${ }^{8}$.

Inclusion $B$. I observed in a thin section of a roughsurfaces specimen of the Orgueil material from the Paris Museum an inclusion of about $1.5 \times 1.0 \mathrm{~mm}$ cross-section. This had an approximately globular habit (in fact, in outline, it had the shape of a jug) and in cross-section appeared to consist of some eight 'bunch-of-grapes'-type 


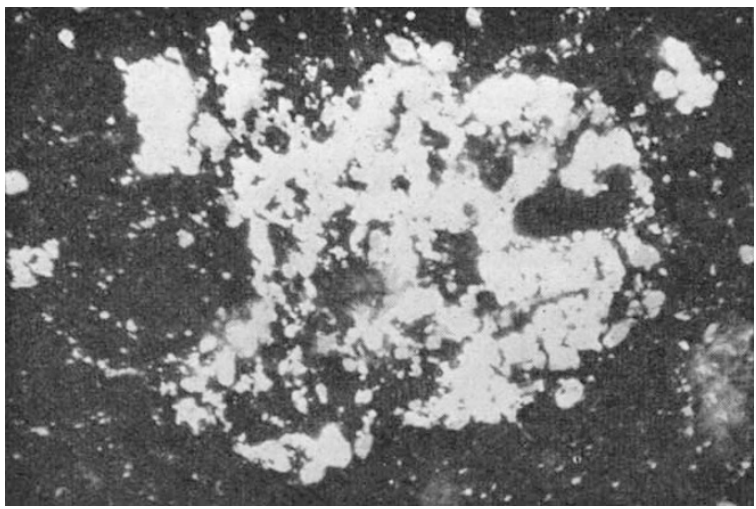

Fig. 1. Group of chondrules forming a small inclusion of "type II' carbonaceous chondrite, within a thin section of Orgueil from the Museum of Paris. The two smaller chondrules on the lower left-hand side of the microphotograph reveal concentrical zoning of transparen (glass and crystalline silicate) and opaque (magnetite, troilite and nickeliron) microchondrules. These iather rare features, however, were also observed by me in the 'type II' Cold Bokkeveldt, and the '

chondrules, and a larger coalescent mass of troilite within the largest of them (Fig. 1). The individual microchondrules were magnetite, troilite, glass and single crystal grains of a material as yet unidentified, but which appeared somewhat corroded and displayed a birefringence similar to that of olivine. The chondrule assemblage within the inclusion was comparable with that of 'type II' stones, in which similar groups of chondrules of up to $10 \mathrm{~mm}$ cross-section frequently appear, without much ground mass.

Inclusion $C$. In the course of dissecting a sample (see inclusion $A$ ), a hard, grey fragment, weighing $0.13 \mathrm{~g}$, was obtained which, so far as could be deduced from the small quantity of substance available, closely resembled the pidgeonite chondrites-this was so both from the point of view of chemical composition (carbon, 0.17 per cent; sulphur, 2.26 per cent; hydrogen, 0.05 per cent), mineral composition and petrographical structure. When a powdered sample of the inclusion was examined, iron-rich olivine and pidgeonite were found together with the usual opaque minerals, including some nickel-iron. These would seem to have originated from major chondri, but a smaller olivine chondrule with the rather unusual feature of a $50 \mu$ diameter gas inclusion was also observed (see ref. 7).

\section{(2) Mighei}

A perfectly angular inclusion of about $4 \mathrm{~cm} \times 2.5 \mathrm{~cm}$ surface area was found in one of the 'type II' Mighei stones from the Moscow Museum. The fragment in question is considerably lighter than the main mass, and an investigation using a binocular microscope revealed a chondrule assemblage characteristic of 'type III' stones.

(3) Sharps

The examples described so far illustrate fragments of meteorites of lower volatiles and lower carbon contents, included in hosts of higher carbon content. In contrast, an approximately brick-shaped 'type I' fragment with well-defined straight boundaries was discovered by Dr. K. Fredriksson (Smithsonian Museum, Washington) in the Sharps meteorite. The meteorite is at present being studied in detail by Dr. Fredriksson, and so this account will be restricted to a few preliminary observations. Both the elemental composition and the petrographical structure of the host material appear to be transitional between 'type III' carbonaceous chondrites and pidgeonite chondrites. The elemental composition of tho inclusion according to Dr. Frodriksson is: carbon, 5.9 per cont; sulphur, 2.5 per cent; water, 2.5 per cent-the percentage of carbon being rather greater, and the sulphur and hydrogen lower than the range of the 'type I' stones at present known. Owing to the oxidizing conditions indicated by the mineral assemblage of the stone (and carbonaceous chondrites in general), it is not surprising that a proportion of the sulphur is found to be in a relatively volatile state as the free element and as sulphates, whereas the bulk of the carbon is in a relativoly non-volatile state as graphite, amorphous carbon and highly polymerized organic molecules, the hydrogen, of course, being volatile. For this reason, we would expect that any thermal metamorphism would have resulted in a depletion of sulphur and hydrogen, without a comparable depletion in carbon; this proved to be the case for the inclusion in question. The high carbon-level suggests that the inclusion was, in its original state, a material even richer in carbon, hydrogen and sulphur than our presently known 'type I' stones. That the inclusion entered the host when the latter was still hot is clearly indicated by the bands of 'redistribution' brought about by distillation of the carbonaceous matter. Such 'redistributions' follow, in a concentrical fashion, the margins of the inclusion and even those of the minor host inclusions or protrusions within the inclusion (see Fig. 2). Such a phase of thermal meta. morphism is also indicated by the rather markedly crystalline nature of the ground mass, and by certain strain

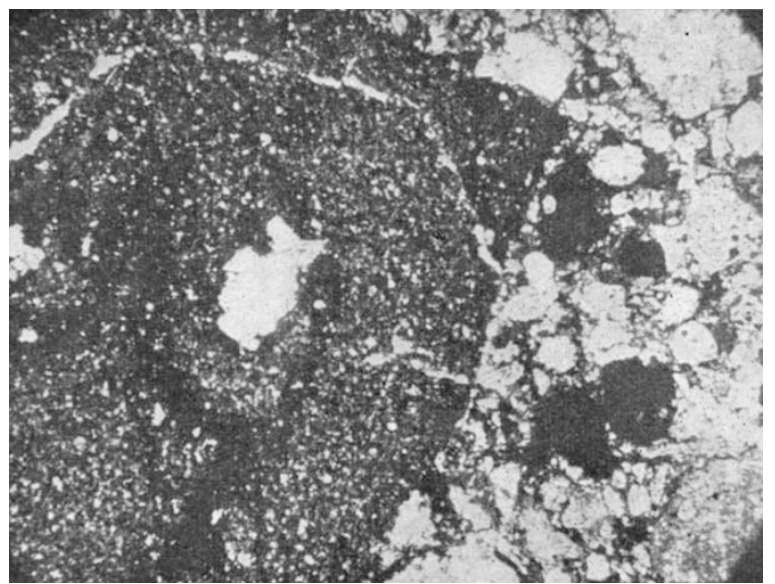

Fig. 2. Part of a 'type 1 ' inclusion from a thin section of Sharps meteorites from the Smithsonian Museum, Washingtion. Note the marked change of statistical petrography between the inclusion and the host, and the ark car the host, within the inclusion proper. Ordinary light. $(\times c, 26)$

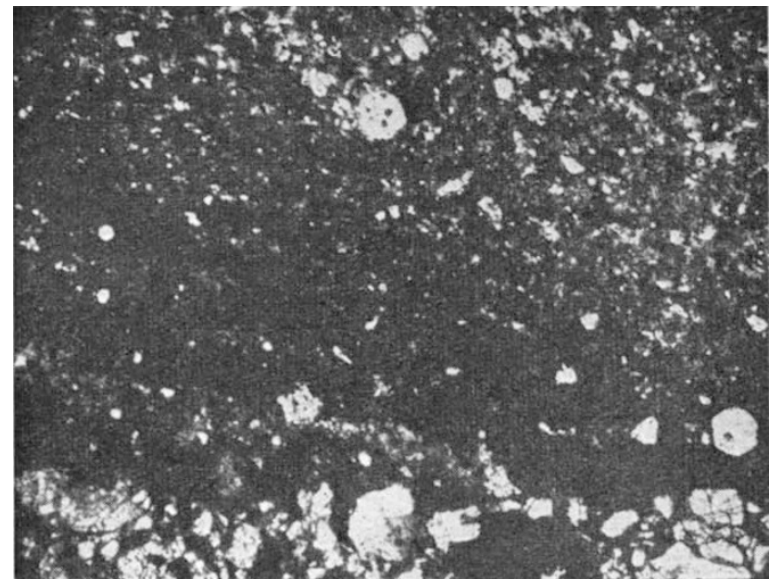

Fig. 3. Detail of an inclusion from Sharps meteorite. The boundary of the host runs along the base of the microphotograph. Note carbonaceous band parallel to the boundary, and also: $(A)$ possibly strained glass cored microchondrule at the bottom right-hand side with a very low (about 0.05 ) diameter of core : diameter of microchondrulc, ratio, which is characteris tic of the 'type I' stones in general (ref. 4); (B) multicore olivine microchondrule, with troilite and nickel-iron cores, at rop centre and ) several glass and crystre. Ordinary light. $(\times 96)$ 
anisotropy of some of the microchondrules of glassy appearance. On the other hand, microchondrules of unstrained glass, and cored microchondrules of crystalline silicate (most likely olivine), do exist (Fig. 3), which makes the similarity between the statistical petrography of 'typo I' stones and the inclusion in question very close indeed.

\section{(4) Renazao}

The features of the inclusion observed in the Sharps meteorite are essentially repeated, within a rather squashed inclusion, with some flow structures, in a thin section of Renazzo from the Smithsonian Museum, Washington (Figs. 4 and 5). The flow structure and inclusions of some chondri in this indicate a more intensive metamorphism, and this impression is strengthened by the more recrystallized nature of the ground mass (compare Figs. 2 and 3 with Figs. 4 and 5). The presence of individual glass and magnetite microchondrules is most significant (Fig. 6) as, in my experience, these are exclusively characteristic of 'type I' stones.

(5) Cold Bokkeveldt

The foregoing examples illustrate associations of meteorites within the chondrite-carbonaceous chondrite

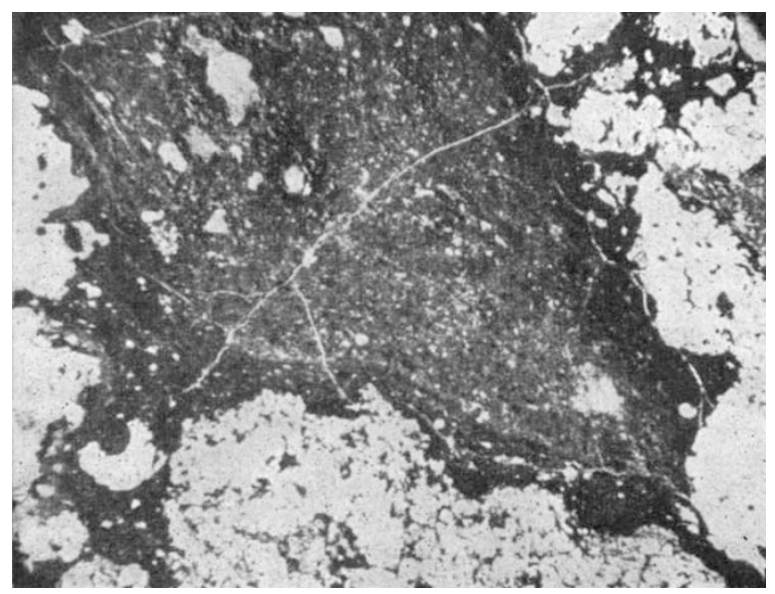

Fig. 4. Inclusion of a 'type $I$ ' stone from a thin section of Renazzo from the Smithsonian Nuseum, Washington. Note indications of extensive thermal metamorphism of inclusion; compared with Sharps meteorite (Fig. 2), there some flow structure is evident, and the mass seems to be partially squeezed in between the gaps of chondrules. Carbon enriched zones are present, similarly to those of Sharps meteorite, parallel to the margins and also surroundirg a group of included chondrules from the host, along the upper left centre of the microphotograph.
Ordinary light. $(\times c .26)$

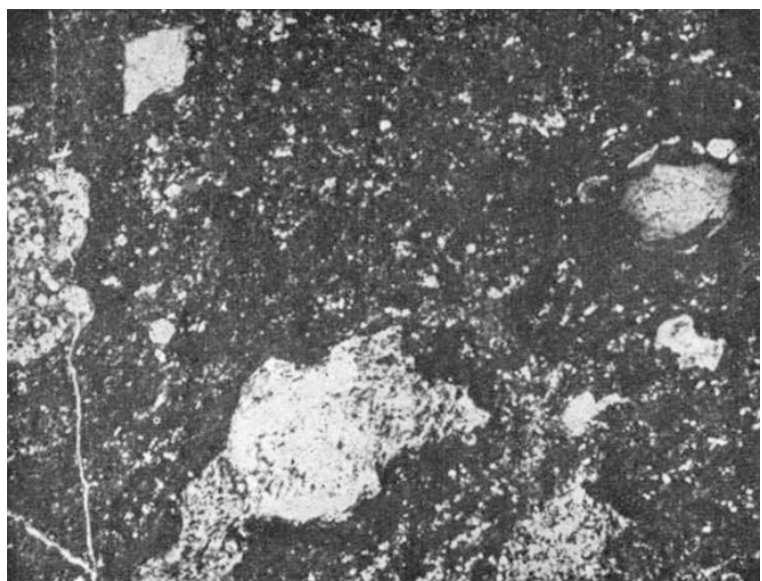

Fig. 5. Detail of the inclusion in Renazzo meteorite shown in Fig. 4. Note almnst continuous opaque (carbonaccous) bands surrounding the two inclusions of the host along left half of the microphotograph, and also large glass microchondrule along right and several small glass
chondri along left centre. Ordinary light. $(\times 96)$

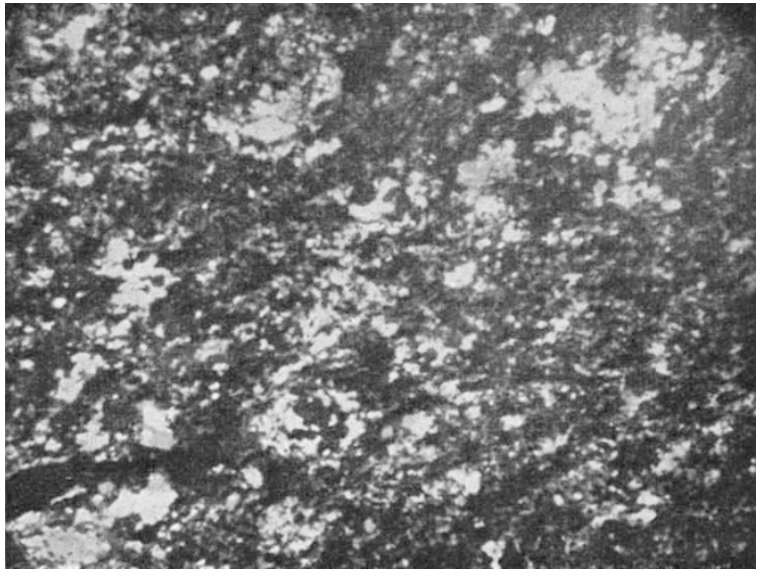

Fig. 6. Ground mass of 'type I' inclusion in Renazzo. Note magnetite microchondrules and groups of partially fused magnetite microchondrules, which, from my previous experience, are quite exclusively $(\times 304$

series. I found in one of the sections of Cold Bokkeveldt meteorite at the British Museum (Natural History) some angular material (perhaps squashed or fragmented large globular type chondrules) reminiscent of typical ureilite structure. An entire section from the same source, however, reveals an exclusively ureilite-type structure with large single crystal grains of clinopyroxene and globular patches of olivine, troilite and nickel-iron, obscured by carbonaecous matter, graphite and possibly diamonds along the boundaries. This structure is characteristic of the Goalpara ureilite, which can be classified as 'type I ureilite'. In the case of Novo Urey and Dyalpur, both the clinopyroxene and the higher melting olivine grains are coalesced into single crystals; these can be classified as 'type II ureilites', as they were formed at higher temperatures. A specimen of Cold Bokkeveldt exhibited in the Museum in Vienna is a breccia revealing numerous angular fragments of dark ureilite in the lighter matrix of the 'type II' stone.

\section{(6) Mokoia}

With the exception of an obvious impact contaminant resin ${ }^{9}$, no inclusions were observed in this meteorite, although Briggs and $\mathrm{Kitto}^{10}$ reported a range of $0 \cdot 5-2 \cdot 1$ per cent carbon in the distinct portions of the samples analysed. The total volatiles of eight different grains from the specimen in the Paris Museum were determined, and a range of between 2.58 per cent and 13.52 per cent was obtained--giving an average of $4 \cdot 36$ per cent. (The standard procedure for determining total volatiles of the stones consisted of heating finely ground powder which had been dried in a vacuum desiccator for $10 \mathrm{~min}$ to $1,000^{\circ}$ $\mathrm{C}$ in a nitrogen atmosphere.) Compared with this, five determinations of total volatiles carried out on the Murray metoorite material only gave a range of $12 \cdot 4-17 \cdot 2$ per cent. It would seem from the results of a minimum of three total volatile determinations that Mokoia may be the most inhomogeneous stone of all those so far investigated, with properties grading from 'type III' to 'type II' within different portions of the stone, even when material is taken from well benoath any known surface erust.

\section{(7) Kainsaz}

The co-existence of chondri and fragmented chondri with partially interlocking aggregates of well-formed olivine and other silicate crystals was noted in the 'type III' Kainsaz and, to a smaller extent, in the 'type II' Cold Bokkeveldt. The latter structures are generally similar to thoso in the chondritic-type achondrites, although major inclusions of achondritic matter have not so far been observed. 


\section{Future Potentialities of the Study of Associations between Diverse Types of Meteorites}

It appears from the literature that research has, on the whole, been restricted to the more obvious cases of markedly brecciated stones. It is possible that much useful information could be obtained through a systematic search for, and study of, the less-evident associations and inhomogeneities, within both the carbonaceous and noncarbonaceous meteorites.

An unexpected example in support of this view became available to me during a recent field study at the village of Barwell, Leicestershire, with members of the University and Museum of Leicester and the British Museum. We were carrying out an investigation of the impact area of the shower of olivine-bronzite chondrites which fell there during December 24, 1965. I had an opportunity there of making a preliminary inspection of about $50 \mathrm{lb}$. of the material. It was observed that individual stones varied in shade from very light to medium dark grey; only further investigations could reveal whether these differences are caused by any variation of the percentage of carbon (which is expected to be within the $0.0 \mathrm{X}$ range for the type of stone in question) or by some other chemical or physical (porosity, etc.) factors. One speci. men contained a globular olivine aggregate, $0 \cdot 5-0 \cdot 75$ in. in diameter, which is unusually large for a chondrule-type structure: certainly it deserves further detailed stuáy.

Such studies could well include an investigation of any chemical interaction between inclusion and host and a search for possible zoning, as well as the chemical and petrographical characterization of 'variable' stones (such as Mokoia and Barwell) which show apparently smooth transitions in properties without any sign of brecciation. In any investigation of these and other problems, the application of some of the more recently developed experimental techniques should prove particularly useful for the detection of changes in the carbonaceous complex-. particularly so spot tests for the distribution of major and minor trace elements, infra-red and ultra-violet spectro. scopy, gas chromatography and mass spectrometry.

\section{Some Theoretical Considerations}

The associations described here clearly point to a common parent body for at least some of the following types of meteorites; (1) Type I-type III carbonaceous chondrites-chondrites (Orgueil, Sharps). (2) Type I-type III carbonaceous chondrite (Renazzo). (3) Type II-type III carbonaceous chondrite (Mighei, Modoia). (4) Type II earbonaceous chondrite-ureilite (Cold Bokkeveldt). (5) Type II carbonaceous chondrite-achondrite (Cold Bokkeveldt). (6) Type III carbonaceous chondrite-achondrite (Kainsaz).

This covers the main range of carbonaceous and noncarbonaceous stony meteorites, with the exception of certain rarer varieties. In view, however, of the very considerable chemical and petrographical uniformity within the 'carbonaceous chondrite-chondrite series', it is likely that, even in cases where associations have not so far been observed in our studies of inclusions, the stones in question originate from identical or very similar parent bodies.

Before any profitable analysis of the possible mechanisms of the emplacements of the fragments can be undertaken, some genetical implications must be considered of the interrelations between volatile and non-volatile constituents of the 'carbonaceous chondrite-chondrite series' (ref. 4). It seems to me that the most likely interpretation of these is that, at the highest temperature stage of its cosmological history, the parent body had an incandescent cloud mantle, covered by cooler clouds of cosmic dust. The 'type I' stones are probably derived from the close-to-surface zones, where mild volcanic activity-pulsation through de-gassing-may have caused a limited amount of intermixing of the cool cosmic dust with spray particles from the marginal zones of the incandescent cloud and volatile condensates. This would explain the small spray diameters and high percentage of glass that was rapidly quenched.

Stores containing less volatile material would be derived from increasingly deeper zones of the parent body, and would have had less access to cosmic dust. They would have received greater quantities of spray particles from the more crowded and hotter portions of the incandescent cloud mantle and, furthermore, would have been subjected to a greater degree of heating subsequent to the final cohesion of the material of the stones from the incandescent clouds. Ureilites, and other types of achondrites, may represent localized spray condensates-that is, 'lavas' - which formed from the highly degassed or impactshocked portions of the incandescent clouds. Alternatively, the features could be explained as being due to a more localized close-to-surface 'ash eruption'-type activity-the stones rich in volatiles coming from the regions farthest from the erupting vent. It is a possibility that the original vertical zoning, through the 'incandescent cloud mantle' model effect, was afterwards modified somewhat by localized volcanic eruptions and intrusions.

The previously observed shift of the volatilization curves towards lower temperatures with the increase of total volatiles content of the stones seems to favour the 'incandescent cloud' model (as discussed in ref. 11). Certain features of the inclusions, however, indicate occasional localized volcanic activity. Thus, the presence of higher volatile, thermally metamorphosed fragments in Sharps and Renazzo can be considered to have arisen from an upsurging column of dense incandescent cloud with perhaps partially solidified spray particles which may have engulfed low-temperature fragments near the surface. The exceptionally large gas bubble in the chondrule of 'Inclusion $C$ ' of Orgueil seems to indicate a sudden release of pressure; it is probable that this was due to the rapid ascent of the fragment in the course of eruptive activity.

The fact that all the inclusions observed so far have welldefined straight boundaries (with the possible exception of inclusion $B$ of Orgeuil) excludes, of course, any possibility of water transportation and is an additional evidence for the essentially 'volcanic breccia'-type origin of the structures. However, in the terrestrial pyroclastics, some rounding of the individual fragments is not uncommon, particularly where more extensive and explosive volcanic activity has occurred. The absence of any sign of rounding, even in such soft material as that included in Sharps, indicates that the volcanic activity was of a mild character and of short duration, as would be expected for a parent body of relatively small dimensions.

Finally, the apparent absence of association between the carbonaceous chondrite and ureilite groups of high carbon stones, on one hand, and the enstatite chondrites, on the other, should be considered. The oxidized iron : reduced iron ratio of carbonaceous chondrites and possibly that of ureilites is the highest in all meteoritic matter. This would suggest that within the close-to-surface zones of the parent body, from which these stones are believed to originate, there was a somewhat hydrogen-depleted, oxidizing assemblage of gases escaping and forming a temporary atmosphere. The high carbon percentage, associated with an extremely low oxidized iron : reduced iron ratio of the enstatite chondrites, may indicate that these stones are derived from the close-to-surface zones of relatively larger parent bodies, in which the de-gassing products, throughout the entire period of de-gassing, remained more reducing as a result of the retention of a higher proportion of hydrogen, methane, and other reducing gases, due to a more intensive gravitational field. If the foregoing considerations are correct, then we should not expect any signs of association between the carbonaceous chondrite-ureilite group of 'oxidized' high volatile stones, and the enstatite chondrite group of 'reduced' ones. It is very likely, however, that a more detailed search would 
reveal interbrecciation between enstatite chondrites of different types.

Finally, the anomalously high carbon : sulphur ratio of $2 \cdot 36$ of the inclusion of Sharps is significant in view of the mean of 0.59 for Type I carbonaceous chondrites. This may be explained as due to preferential escape in the course of the thermal metamorphism of the fragment of sulphur as sulphur vapour and sulphur dioxide, and the retention of amorphous carbon and graphite under the somewhat oxidizing conditions that prevailed. The even higher carbon: sulphur ratio for the ureilites of about $6 \cdot 0$ can be explained by the same mechanism, if more intensive heating is postulated: in this connexion analytical data regarding the ureilite-type fragments in Cold Bokkeveldt would be of considerable interest. On the other hand, de-volatilization under more reducing conditions would cause essentially the reverse trend, as then the bulk of sulphur is present as non-volatile ferrous sulphide and the carbon forms mainly volatile hydrocarbons. It is possible that the latter explanation would account for the low sulphur : carbon ratio of 0.06 for the enstatite chondrites and the even lower value (of the order of 0.01 ) for the ordinary chondrites.

These observations and considerations would seem to indicate that the very broad range of sulphur : carbon ratios of meteorites can be derived through de-volatilization of primordial matter (cosmic dust) of approximately uniform composition under differing temperatures and reducing or oxidizing conditions. Detailed investigations of thecomposition of inclusions of carbon, sulphur and other volatile elements or inclusions may give us further signicant information in assessing the relative importance of the parameters, including the time involved.

I would like to thank Prof. J. D. Bernal, Birkbeck College, London (where much of the work was actually carried out), for encouragement, and Prof. J. Orcel (Paris), Prof. K. Strokay (Budapest), Dr. Henderson and Dr. B. Mason (Smithsonian Institution, Washington), Dr. H. Scholler (Museum of Vienna), Dr. H. B. Wiik (Helsinki), and Dr. G. F. Claringbull (British Museum (Natural History)) for specimens and the opportunity to study thin sections. I also thank Dr. K. Fredriksson (Smithsonian Institution, Washington) for information about his discovery of the inclusion in the thin sections in Sharps meteorite and for placing these at my disposal.

Department of Geology,

University of Concepcion,

$$
\text { Chile. }
$$

${ }^{1}$ Wood, J. A., in The Moon, Meteorites and Comets, edit. by Middlehurst and Kuiper, 337 (The University of Chicago Press, 1963).

${ }^{2}$ Anders, E., Space Sci. Rev., 3, 583 (1964).

'Wilk, H. B., Geochim. Cosmochim. Acta, 9, 279 (1956).

Wik, H. B., Geochim. Cosmochim. Acta, 9, $279(1956)$.
Mress, 1963), also unpublished results.

${ }^{5}$ Mueller, G., Nature, 196, 929 (1962).

'Mason, B., Space Sci. Rev., 1, 621 (1962).

${ }^{7}$ Mueller, G., Adv. Org. Geochem., Pergamon Press, 112 (1963)

Bostrom, K., and Fredriksson, K., pre-print for publications from The Smithsonian Institution, Washington (1965).
9 Mueller, G., Nature, 204, 567 (1964).

10 Briggs, M. H., and Kitto, G. B., Nature, 193, 1130 (1962).

${ }_{11}$ Mueller, G., Shaw, R. A., and Takeshi, Ogava, Nature, 206, 23 (1965).

I CONSIDER the studies contained in Prof. Mueller's article to be of great importance. Much has been shown by examining the single meteorite stone, but this work, for the most part, has consisted of investigations on the stone as a whole attributed to various classes. What Prof. Mueller has done in this way is to examine meteorites containing other types of meteorite as inclusions, thus demonstrating that they must have been derived from the same parent body.

I would like to direct particular attention to the study, by Dr. Fredriksson, of the Sharps meteorite and its bearing on the origin of carbonaceous meteorites 'type I'. At present, only preliminary observations have been made: in my opinion their bearing is conclusive; the Sharps meteorite contains at least one large inclusion of a 'type I' carbonaceous stone.

Two features of this observation call for particular attention. First, the inclusion is an angular fragment with generally defined straight boundaries against the surrounding chondrite. This indicates that it is a fragment broken from a larger, already formed, carbonaceous meteorite which has been incorporated, subsequent to its breaking up, in the then unconsolidated chondrite stone. Secondly, the fragment, as it is found, shows evidence of contact metamorphism, presumably in bands following the outline of the fragment as well as those of the included chondrules. This shows that at the time of inclusion the chondrules were hotter than the carbonaceous material although not so hot as to cause excessive degassing or boiling away of the volatile constituents.

It is clear that further investigation would throw much light on the origin of both types of meteorite. Prof. Mueller has put forward plausible accounts of these with which I am inclined to agree, but more may come out of a more detailed study. This should include a micro-chemical analysis point by point both inside and outside the inclusion to check how far the material had been altered and how far the contact metamorphism has affected the parent body. For this, electron probe investigations might be very useful and also electron spin resonance and nuclear magnetic resonance.

Some such studies will undoubtedly be carried out and it would be appropriate for much of it to be done in Britain, for the British Museum (Natural History) holds the most extensive collection of meteorites in the world. The personnel and apparatus do not yet exist in Britain for such studies to be carried out on an adequate scale. Unless such resources and manpower can be made available, a field of great promise in the age of spacial exploration of our planetary system could well be lost.

Department of Crystallography, J. D. Bernal Birkbeck College, London, W.C.1.

\title{
ESTIMATION OF ZINC-65 BACKGROUND LEVELS FOR MARINE COASTAL WATERS
}

\author{
By G. V. ALEXANDER and R. H. ROWLAND \\ Laboratory of Nuclear Medicine and Radiation Biology of the Department of Biophysics and Nuclear Medicine, \\ School of Medicine, University of California at Los Angeles
}

$\mathrm{W}$ ITH the increased possibility of a nuclear accident near or in the marine coastal environment a more accurate estimate of background radioactivity levels for elements such as zinc-65 is needed. Estimates of zinc-65 on the Pacific coast are complicated by the uncertainty of the contribution resulting from the Columbia
River outflow. The average rate of input from this source is $30 \mathrm{c}$. zinc-65 and $700 \mathrm{c}$. chromium-51 per day ${ }^{2}$. Even at these relatively high levels dilution with sea water soon reduces the concentration to such a degree that direct measurement in sea water is impossible. Chemical concentration of zinc-65 in sea-water samples has generally 